\title{
Diseño y cotización de una planta de tratamiento de aguas residuales para parroquias rurales del Cantón Riobamba - Provincia de Chimborazo-Ecuador
}

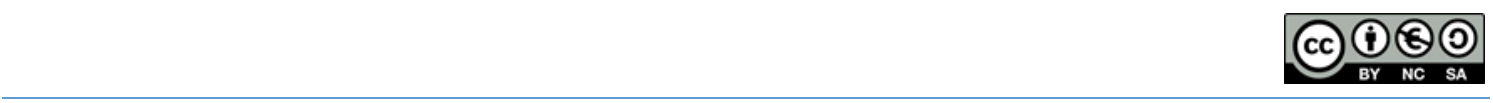

Design and quotation of a wastewater treatment plant for rural parishes of the Riobamba Canton- Chimborazo Province-Ecuador

Mónica Lilian Andrade Avalos. ${ }^{1}$, Danielita Fernanda Borja Mayorga. ${ }^{2}$, \& Marlene Jacqueline García Veloz. ${ }^{3}$

\begin{abstract}
.
Introduction. Construction and maintenance costs represent a primary aspect in the design and construction of wastewater treatment plants. Objetives. Design and quote a wastewater treatment plant in the rural area of Riobamba canton, taking the Santiago de Calpi Parish Head as a point of analysis to avoid contamination to the Chibunga River. Methodology. Compound sampling was used for the characterization of wastewater, it was shown that Fecal Coliforms, Suspended Solids, Surfactants, Oils and Fats, Biochemical Oxygen Demand $\left(\mathrm{DBO}_{5}\right)$, Chemical Oxygen Demand (DQO) and Turbidity were the parameters that were consider for the elaboration of the plant design, data compared with the regulations of the Unified Text of Secondary Environmental Legislation of the Ministry of the Environment (TULSMA). Results. The treatment plant is projected for 20 years for a population of 1,887 inhabitants and with a flow of $1,479.6 \mathrm{~m} 3$ / day. The system consists of a channel, manual cleaning grating system, a

1 Facultad de Ingeniería Química, Escuela Politécnica Superior de Chimborazo, Riobamba-Ecuador, moandrade@espoch.edu.ec, orcid.org/0000-0001-5736-5607

2 Facultad de Ingeniería Química, Escuela Politécnica Superior de Chimborazo, Riobamba-Ecuador, dborja@espoch.edu.ec, orcid.org/0000-0002-8438-064X

3 Facultad de Ingeniería Química, Escuela Politécnica Superior de Chimborazo, Riobamba-Ecuador, marlene.garcia@espoch.edu.ec, orcid.org/0000-0002-9996-1594
\end{abstract}


primary settler, an activated sludge tank that goes together with a secondary settler, a disinfection tank, it should be emphasized that the sludge generated in the settlers needs treatment and is proposes 4 drying beds. When carrying out this purification process, results are obtained with a $\mathrm{BOD}_{5} 37 \mathrm{mg} / \mathrm{L}, \mathrm{COD} 59 \mathrm{mg} / \mathrm{L}$, Suspended Solids $2 \mathrm{mg} /$ L, Surfactants $0.4 \mathrm{mg} / \mathrm{L}$, Oils and Fats $0.2 \mathrm{mg} / \mathrm{L}$ and Coliforms fecal $<2 \mathrm{CFU} /$ $100 \mathrm{ml}$, these results are in accordance with the TULSMA, book VI, Annex 1, table 9 Discharge into a body of Fresh Water. Conclusion. The total price of the wastewater treatment plant is $\$ 31699,40$ considering good quality materials for its construction, extending the useful life of the equipment and of the plant.

Keywords: Wastewater treatment/ Pollutant load/ Permissible limits/ Quotation/ Activated sludge.

\section{Resumen.}

Introducción. Los costos de construcción y mantenimiento representan un aspecto primordial en el diseño y construcción de plantas de tratamiento de aguas residuales. Objetivo. Diseñar y cotizar una planta de tratamiento de aguas residuales en la zona rural del cantón Riobamba, tomando como punto de análisis la Cabecera Parroquial Santiago de Calpi para evitar la contaminación al Río Chibunga. Metodología. Se utilizó el muestreo compuesto para la caracterización de las aguas residuales se demostró que los Coliformes Fecales, Sólidos Suspendidos, Tensoactivos, Aceites y Grasas, Demanda Bioquímica de Oxígeno $\left(\mathrm{DBO}_{5}\right)$, Demanda Química de Oxígeno (DQO) y Turbidez fueron los parámetros que se consideran para la elaboración del diseño de la planta, datos comparados con la normativa del Texto Unificado de Legislación Ambiental Secundaria del Ministerio del Ambiente (TULSMA). Resultados. La planta de tratamiento está proyectada a 20 años para una población de 1887 habitantes y con un caudal de 1479,6 m²/día. El sistema consta de un canal, sistema de rejillas de limpieza manual, un sedimentador primario, un tanque de lodos activados que va conjuntamente con un sedimentador secundario, un tanque de desinfección, cabe recalcar que los lodos generados en los sedimentadores necesitan un tratamiento y se propone 4 lechos de secado. Al realizar este proceso de depuración se logra obtener resultados con una $\mathrm{DBO}_{5} 37 \mathrm{mg} / \mathrm{L}$, DQO $59 \mathrm{mg} / \mathrm{L}$, Sólidos Suspendidos 2 $\mathrm{mg} / \mathrm{L}$, Tensoactivos $0,4 \mathrm{mg} / \mathrm{L}$, Aceites y Grasas $0,2 \mathrm{mg} / \mathrm{L}$ y Coliformes fecales $<2$ UFC/100ml dichos resultados están acorde con el TULSMA, libro VI, Anexo 1, tabla 9 - Descarga a un cuerpo de Agua Dulce. Conclusión. La cotización total de la planta de tratamiento de aguas residuales es de $\$ 31699,40$ considerando materiales de buena calidad para su construcción ampliando la vida útil de los equipos y de la planta.

Palabras claves: Tratamiento de aguas residuales/ Carga contaminante/ Límites permisibles/ Cotización/ Lodos activados. 


\section{Introducción.}

El agua es un recurso muy importante para el desarrollo de actividades del hombre en las distintas áreas industriales así como para satisfacer necesidades personales. El efluente de estas actividades es descargado sin un previo tratamiento esto produce impactos ambientales negativos en los cursos de agua receptores, debido a la concentración de contaminantes que dichas aguas contengan.

Estos efluentes no pueden ser desechados directamente al medio ambiente sin recibir un tratamiento con la finalidad de reducir problemas graves al ecosistema. Este tipo de residuos se generan en la utilización del agua en procesos domésticos o industriales, los cuales arrastran diferentes tipos de residuos de materia orgánica e inorgánica.

Se debe devolver sustancias al agua por debajo de las concentraciones límites, se inicia un proceso de autodepuración, debido a diversos microorganismos. Estos microorganismos descomponen los desechos, metabolizándolos y transformándolos en sustancias simples, como dióxido de carbono, nitrógeno, etc. Este proceso se aplica a sustancias orgánicas, como detergentes y fenoles, y también a algunas sustancias inorgánicas, ya que hay microorganismos capaces de absorber ciertos metales, incorporándolos a sus células.

Las sustancias arrojadas que poseen una alta concentración de materias tóxicas, provocan que los microorganismos sean destruidos y se anula la autodepuración. Además pueden morir organismos más grandes, como peces, crustáceos, y plantas acuáticas, por intoxicación o por falta de microorganismos para alimentarse. Estos a su vez pueden intoxicar al resto de la fauna que conforma la cadena alimentaria, eventualmente llegando hasta el hombre.

La descarga de estas aguas ha causado la generación de distintos tipos de contaminantes como: malos olores, enfermedades, sedimentos, bacterias, etc. El tratamiento a estas aguas residuales se da con la eliminación de microorganismos patógenos; así evitando que lleguen a contaminar vertientes cercanas del lugar de desecho. Mediante el estudio e investigación de estos efluentes se puede realizar un tratamiento que se está implementando en todos los países del mundo; permitiendo así que estos efluentes puedan ser reutilizados para riego de cultivos, crianza de animales e incluso para el consumo humano.

Todo este proceso se realiza con el fin de dar una mejor calidad de vida a los a la sociedad y cumpliendo con lo que dicta la norma (TULSMA) de calidad del medio ambiente y descarga de efluentes Libro VI, Anexo 1, Tabla 9.

La parroquia rural Santiago de Calpi del Cantón Riobamba de la provincia de Chimborazo, se encuentra entre las más antiguas de Ecuador, la parroquia tiene 19 comunidades, lo que representa alrededor de 18.000 personas. La población se compone 
de indígenas y campesinos en su mayoría. La población es joven, siguiendo las mismas cifras que a nivel nacional, (49,5\% de la población tiene menos de 20 años).

La población que presenta la cabecera cantonal de Santiago de Calpi es de 698 representando 8,86\% del total de la Parroquia, este dato obtenido en el último CENSO del 2010. Alrededor del $93 \%$ de los habitantes tienen sus propias viviendas, sus pequeños terrenos y algunos animales. La base de la economía del campesino es la producción agrícola, las viviendas en su mayoría son hechas de fábrica (bloque, ladrillos). Disponiendo del servicio de agua entubada (no es potable) para el consumo. Pocas viviendas cuentan con letrina. Los caminos son en tierra y deterioran cuando llueve.

La Parroquia Santiago de Calpi se ha visto con la necesidad de realizar un "Diseño y cotización de una planta de tratamiento de aguas residuales" por los diferentes problemas que está causando esta descarga directa al Río Chibunga, la misma que genera enfermedades a las comunidades que utilizan éste recurso. Enfermedades como cólera, amebiasis, gardiasis entre otras son los causantes de que la población este con problemas de este tipo.

Otro agente perjudicado son los cultivos que posee esta Parroquia, estas aguas al ser utilizadas en el riego están causando la disminución en la producción de los variados sembríos de la zona como cultivos de papa, mellocos, choclos, etc. Los cuales están muy cercanos a la fuente de desembocadura y estos poseen residuos de fertilizantes, pesticidas provocando un impacto ambiental a fuentes cercanas del mismo.

Cerca del depósito de estas aguas se encuentra una lubricadora de autos, la cual genera diferentes tipos de residuos de hidrocarburos los cuales son desechados directamente a las tuberías de descarga de estas aguas.

En la zona existen grandes cultivos de papa, mellocos, choclos, etc. Los cuales están muy cercanos a la fuente de desembocadura y estos poseen residuos de fertilizantes, pesticidas provocando un impacto ambiental a fuentes cercanas del mismo.

En esta investigación se busca diseñar y cotizar un sistema de tratamiento de aguas residuales, el mismo que consiste en la caracterización físico-química y microbiológica del efluente siguiendo los diferentes métodos de determinación, medición del caudal que genera la población, el estudio del proyecto se busca recolectar la información necesaria para el realizar el diseño de la planta de tratamiento brindando una eficiencia y accesibilidad económica de la misma. 


\section{Metodologia.}

\section{Material y Métodos}

\section{Selección de la muestra}

El tipo de muestreo que se utilizó en esta investigación es el muestreo compuesto el cual varia por su punto de descarga, hora formando una mezcla homogénea por día se tuvo la muestra compuesta. El plan de muestreo se determinó mediante el caudal; se tomó por una semana (siete días), brindando diferentes tipos de datos de concentración por día y hora. Mediante la medición del caudal se pudo determinar la muestra representativa para realizar la primera caracterización en el laboratorio. Se obtuvo tres análisis de caracterización de las muestras, tomadas de diferentes días y horas formando la muestra compuesta. El volumen tomado para la caracterización del análisis del laboratorio fue de 6 litros tomando en cuenta que esta ya es una muestra compuesta cada 10 minutos en el lugar del muestreo.

Todos los procesos de laboratorio realizados para obtener el valor de cada uno de los parámetros fueron realizados de acuerdo al manual de procedimiento técnico del Laboratorio de Aguas de la Facultad de Ciencias - ESPOCH.

Se realizaron análisis físico-químicos y microbiológicos del agua residual, y los resultados se compararon con TULSMA, Anexo 1, Libro IV, tabla 9 Límites de descarga a un cuerpo los resultados se aprecian en la Tabla.

TABLA 1: Parámetros analizados fuera de los límites permisibles

\begin{tabular}{llll}
\hline Parámetros & Unidad & Resultado & $\begin{array}{l}\text { Valor } \\
\text { limite }\end{array}$ \\
\hline Tensoactivos & $\mathrm{mg} / \mathrm{l}$ & 2,70 & 0,5 \\
$\begin{array}{l}\text { Aceites y grasas } \\
\begin{array}{l}\text { Demanda Química de } \\
\text { Oxígeno (DQO) }\end{array}\end{array}$ & 28 & 0,5 \\
$\begin{array}{l}\text { Demanda Bioquímica } \\
\text { de oxígeno (DBO5) }\end{array}$ & 368,33 & 200 \\
$\begin{array}{l}\text { Sólidos Suspendidos } \\
\text { Mg/l }\end{array}$ & 287,66 & 100 \\
\hline
\end{tabular}

Fuete: Grupo Investigación Facultad Ciencias, 2015

Una vez obtenidos los resultados de la caracterización se obtiene el índice de biodegradabilidad para conocer qué tipo de tratamiento es factible aplicar para el agua residual. 


$$
\begin{gathered}
\frac{D B O 5}{D Q O}=\frac{287,66}{368,33}=0,78 \\
0,78>0.24 \text { (muy biodegradable) }
\end{gathered}
$$

El resultado nos indica que es muy biodegradable (Tabla 2) y por lo tanto se debe aplicar sistemas de lodos activados para su tratamiento.

TABLA 2: Índice de biodegradabilidad

\begin{tabular}{cc}
\hline $\mathbf{D B O}_{5} / \mathbf{D Q O}$ & Índice de biodegradabilidad \\
\hline$<0,25$ & No biodegradable \\
$0,25-0,4$ & Biodegradable \\
$>0,4$ & Muy biodegradable \\
\hline
\end{tabular}

Fuente Morante Hilda (Morante, 2011)

\section{Diseño del sistema de tratamientos de agua residual}

El diseño y capacidad de los equipos se debe fundamentar en dos puntos importantes: primero en el caudal de diseño del sistema de tratamiento de aguas residuales y en la utilización de sistemas de lodos activados por su alta eficiencia para la remoción de la materia orgánica en procesos de tratamientos ambientales.

\section{Determinación del caudal del agua residual}

Para la determinación del caudal se ocupó un balde graduado de 8L durante los 7 días de la semana empezando a las 7:00 am hasta las 19:00 pm cada día. Para la medición del caudal se realizó la toma de muestras cada media hora.

Le método utilizado para la medición del caudal fue la del llenado del recipiente para caudales de bajo volumen donde se llega a determinar que el caudal varía según la hora de la muestra tomada.Una vez obtenidos los datos se realiza los cálculos pertinentes para determinar el caudal promedio semanal como se indica en la Tabla 3.

\begin{tabular}{|c|c|c|c|c|}
\hline \multirow[t]{2}{*}{ DÍA } & \multirow{2}{*}{$\begin{array}{l}\text { CAUDAL } \\
\mathbf{Q}(\mathrm{L} / \mathrm{s})\end{array}$} & \multirow{2}{*}{$\begin{array}{l}\text { CAUDAL } \\
\text { Q (m3/h) }\end{array}$} & CAUDAL & CAUDAL \\
\hline & & & $\begin{array}{l}\text { Q MAX } \\
(\mathrm{L} / \mathbf{s})\end{array}$ & $\begin{array}{l}Q \quad \text { MAX } \\
(\mathrm{m} 3 / \mathrm{h})\end{array}$ \\
\hline Lunes & 1,3575 & 4,887 & 1,946 & 6,698 \\
\hline Martes & 1,3703 & 4,933 & 1,86 & 6,698 \\
\hline Miércoles & 1,3387 & 4,8192 & 1,689 & 6,082 \\
\hline Jueves & 1,3991 & 5,0368 & 1,781 & 6,412 \\
\hline Viernes & 1,3463 & 4,8467 & 1,968 & 7,068 \\
\hline Sábado & 1,3762 & 4,9545 & 1,849 & 6,656 \\
\hline Domingo & 1,35 & 4,8598 & 1,889 & 6,799 \\
\hline
\end{tabular}

TABLA 3: Promedio semanal del caudal de agua residual 


\begin{tabular}{lllll}
\hline $\boldsymbol{\Sigma}$ & 9,5381 & 34,337 & 12,982 & 46,413 \\
Promedio & 1,3626 & 4,9053 & 1,8546 & 6,6304
\end{tabular}

FUENTE: Grupo Investigación Facultad Ciencias, 2017

\section{Sistema de lodos activados}

Es un tratamiento bilógico de aguas residuales que emplea microorganismos aerobios y facultativos para poder retirar la materia orgánica presente en el agua residual. Engloba un proceso de limpieza física, química y biológica (Geocities 2009)

Este método de tratamiento sintetiza la carga microbiana interviniendo en el balance de materia mediante un reactor bilógico.

Por lo general el método de lodos activados se lo realiza de conjuntamente con una sedimentación secundaria el cual ayuda que los sólidos suspendidos en el agua residual sean eliminados en el sedimentador secundario en ocasiones se realiza una recirculación del agua al tanque aireador ayudando a que el proceso sea más eficiente, puede ser de flujo continuo o de mezcla completa y este reactor de lodos activados se utiliza con una bomba o compresor el cual dará una potencia requerida para el proceso.

La aireación que debe recibir el agua residual varía de 4 a 8 horas dependiendo la carga orgánica presente en el agua.

El sistema de lodos activados es proceso continuo del tanque sedimentador primario, dicho reactor está compuesto por difusores y un sedimentador secundario, sistema que no necesita recirculación de lodos para el tratamiento biológico.

\section{Sistema de Tratamientos de Agua Residual}

\section{GRÁFICO 1. Diagrama de flujo del sistema de tratamiento de agua residual}

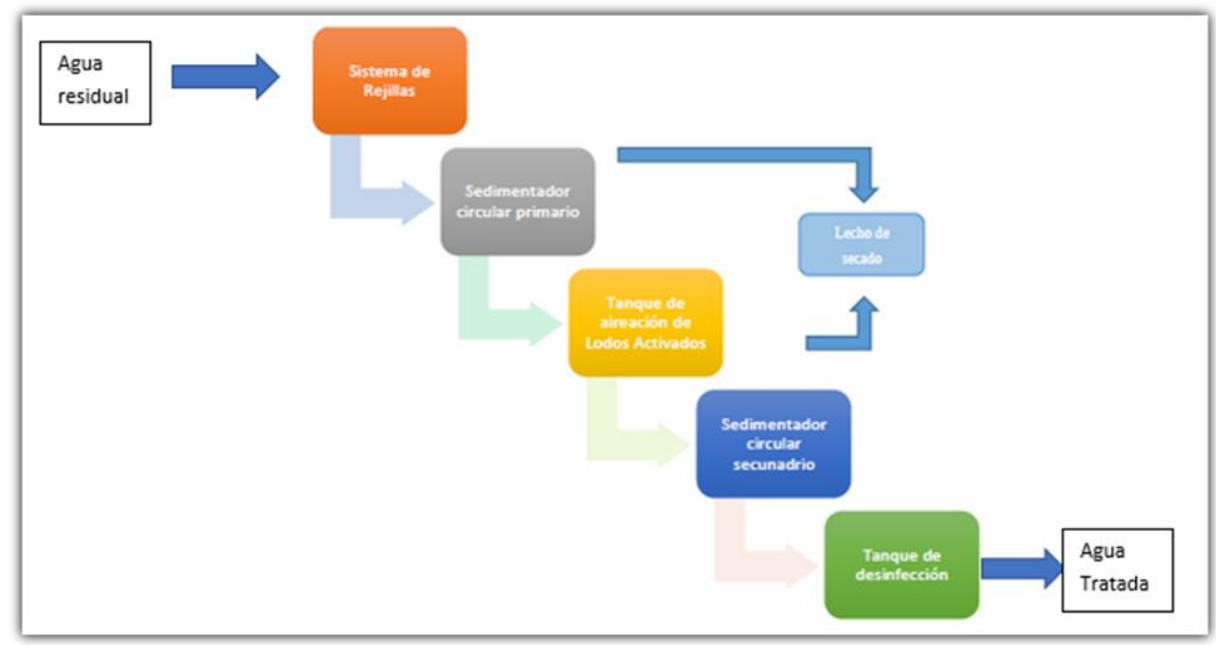

FUENTE: Grupo Investigación Facultad Ciencias, 2015 
TABLA 4: Caudal de diseño

\begin{tabular}{cc}
\hline Parámetro & Resultado \\
Dotación & $120 \mathrm{~L} / \mathrm{s}$ \\
Caudal de diseño & $17,125 \mathrm{~L} / \mathrm{s}$ \\
Caudal medio de aguas residuales & $1,834 \mathrm{~L} / \mathrm{s}$ \\
Caudal medio diario & $12,454 \mathrm{~L} / \mathrm{s}$ \\
\hline FUENTE: Grupo Investigación Facultad Ciencias, 2015
\end{tabular}

TABLA 5: Diseño de Rejillas

\begin{tabular}{cc}
\hline Parámetro & Dimensión \\
$* \mathbf{B}$ & 1,79 \\
**Coeficiente de Manning & 0,013 \\
Ancho & $0,5 \mathrm{~m}$ \\
Profundidad & $0,5 \mathrm{~m}$ \\
Separación entre barras & $0,025 \mathrm{~m}$ \\
Pendiente en relación con la & \\
vertical & $45^{\circ}$ \\
Velocidad de aproximación & $0,45 \mathrm{~m} / \mathrm{s}$ \\
Espesor de la barra & $0,010 \mathrm{~m}$ \\
Tipo de barra circular & 1,79 \\
Longitud de las barras & 0,82 \\
Número de barras & 14 \\
Nivel máximo de agua & $0,0761 \mathrm{~m}$ \\
Longitud sumergida de la varilla & $0,107 \mathrm{~m}$ \\
Pérdida de carga de las rejillas & $0,00385 \mathrm{~m}$ \\
\hline
\end{tabular}

FUENTE: Grupo Investigación Facultad Ciencias, 2015 
TABLA 6: Sedimentador primario

\author{
Parámetro \\ *Velocidad terminal \\ **Constante del material \\ arrastrado
}

Área

Ancho

Largo

Altura

Volumen

Diámetro

Tiempo de retención hidráulica

Área de la sección Transversal

Velocidad de arrastre

Porcentaje de remoción de DBO5

Porcentaje de remoción de SS

*** Valores empíricos

\section{Dimensión}

$3 \mathrm{~m} / \mathrm{h}$

0,05

$20,55 \mathrm{~m}^{2}$

$3,21 \mathrm{~m}$

$6,40 \mathrm{~m}$

$3,6 \mathrm{~m}$

$73,96 \mathrm{~m}^{3}$

$5,115 \mathrm{~m}$

$1,20 \mathrm{~h}$

$11,56 \mathrm{~m}$

$0,0626 \mathrm{~m} / \mathrm{s}$

$28,57 \%$

$49,38 \%$

$\mathrm{a}=0,018$

$\mathrm{b}=0,02$

Fuente: *AURELIO HERNANDEZ, ** Manual de depuración Urbana., p. 96, METCALF \& EDDY., Ingeniería de Aguas Residuales., 1995., ${ }^{* * *}$ CRITES, R., Tratamiento de aguas residuales en pequeñas poblaciones

TABLA 7: Sistema de lodos activados

\begin{tabular}{|c|c|}
\hline Parámetro & Dimensión \\
\hline Caudal tratante del reactor & 1479,6 m³/día \\
\hline **Tiempo de retención celular & 10 días \\
\hline **Coeficiente de producción celular & $\begin{array}{c}0,60 \mathrm{Kg} \text { de células producidas / Kg de } \\
\text { materia orgánica }\end{array}$ \\
\hline $\begin{array}{l}\text { **Concentración de sólidos suspendidos } \\
\text { volátiles en el tanque de aireación| }\end{array}$ & $4 \mathrm{Kg} / \mathrm{m}^{3}$ \\
\hline
\end{tabular}




$\begin{array}{cc}\text { *Coeficiente de degradación endógena } & 0,06 \mathrm{día}^{-1} \\ \text { *Concentración de DBO del afluente } & 0,288 \mathrm{Kg} / \mathrm{m}^{3} \\ \text { *Concentración del DBO del efluente } & 0,038 \mathrm{Kg} / \mathrm{m}^{3} \\ \text { Eficiencia basada en el DBO5 soluble } & 86,68 \% \\ \text { Volumen del reactor } & 345,81 \mathrm{~m}^{3} \\ \text { Tiempo de retención hidráulico } & 5,61 \mathrm{~h} \\ \text { Relación } & 0,312 \mathrm{día}^{-1} \\ \text { Alimento/microorganismo } & \\ \text { Producción de lodo observada } & \mathrm{de} \text { materia orgánica } \\ \text { Producción diaria de fango } & 138,32 \mathrm{Kg} / \mathrm{día} \\ \text { Caudal de lodo de purga } & 34,58 \mathrm{~m} 3 / \text { día } \\ \text { Necesidad de oxígeno } & 541,31 \mathrm{Kg} \mathrm{O} / \mathrm{día} \\ \text { Potencia necesaria por los difusores } & 45 \mathrm{KW} \\ \text { Largo } & 12 \mathrm{~m} \\ \text { Ancho } & 10 \mathrm{~m} \\ \text { Altura } & 3 \mathrm{~m}\end{array}$

Fuente: * Grupo Investigación Facultad Ciencias, 2015., **METCALF \& EDDY., Ingeniería de Aguas Residuales., 1995

TABLA 8: Sedimentador secundario

\begin{tabular}{cc}
\hline Parámetro & Dimensión \\
\hline *arga superficial & $49 \mathrm{~m}^{3} / \mathrm{m}^{2}$ día \\
*Profundidad & $3,5 \mathrm{~m}$ \\
Área & $30,19 \mathrm{~m}^{2}$ \\
Diámetro & $6,2 \mathrm{~m}$ \\
Reparto Central & $1,55 \mathrm{~m}$ \\
Altura del reparto & $1 \mathrm{~m}$ \\
Carga de vertedero & $0,000879 \mathrm{~m}^{2} / \mathrm{s}$ \\
Ancho & $3,88 \mathrm{~m}$ \\
Largo & $7,77 \mathrm{~m}$
\end{tabular}




\section{Volumen}

Tiempo de retención hidráulica

Remoción del $\mathrm{DBO}_{5}$

Remoción de sólidos suspendidos
$108,53 \mathrm{~m}^{3}$

$1,76 \mathrm{~h}$

$33,08 \%$

$54,76 \%$

Fuente: *METCALF \& EDDY., Ingeniería de Aguas Residuales., 1995

TABLA 9: Tanque de desinfección

\begin{tabular}{cc}
\hline Parámetro & Dimensión \\
\hline Peso requerido de desinfectante & $2,22 \mathrm{Kg} /$ día \\
Volumen del tanque de cloración & $0,76 \mathrm{~m}^{3}$ \\
Volumen del tanque de desinfección & $30,82 \mathrm{~m}^{3}$ \\
Largo del tanque & $3,45 \mathrm{~m}$ \\
Ancho del tanque & $3 \mathrm{~m}$ \\
Altura del tanque & 2,99 \\
\hline
\end{tabular}

Fuente: Grupo Investigación Facultad Ciencias, 2015

TABLA 10: Lecho de secado

\begin{tabular}{cc}
\hline Parámetro & Dimensión \\
\hline Carga de sólidos & $288,522 \mathrm{Kg} /$ día \\
Masa de sólidos & $93,769 \mathrm{Kg} / \mathrm{día}$ \\
Volumen diario de lodos & $751,359 \mathrm{~L} / \mathrm{día}$ \\
Área del secador & $75,125 \mathrm{~m}^{2}$ \\
Número de lechos de secado & 4 \\
*Base & $3 \mathrm{~m}$ \\
Longitud & $6,26 \mathrm{~m}$ \\
*Profundidad & $0,40 \mathrm{~m}$ \\
\hline Fuente: Grupo Investigación Facultad Ciencias, 2015, * Normas Ecuatorianas INEN
\end{tabular}


GRÁFICO 2. Diseño de una Planta de Tratamiento de A.R. para la Cabecera Parroquial Santiago de Calpi

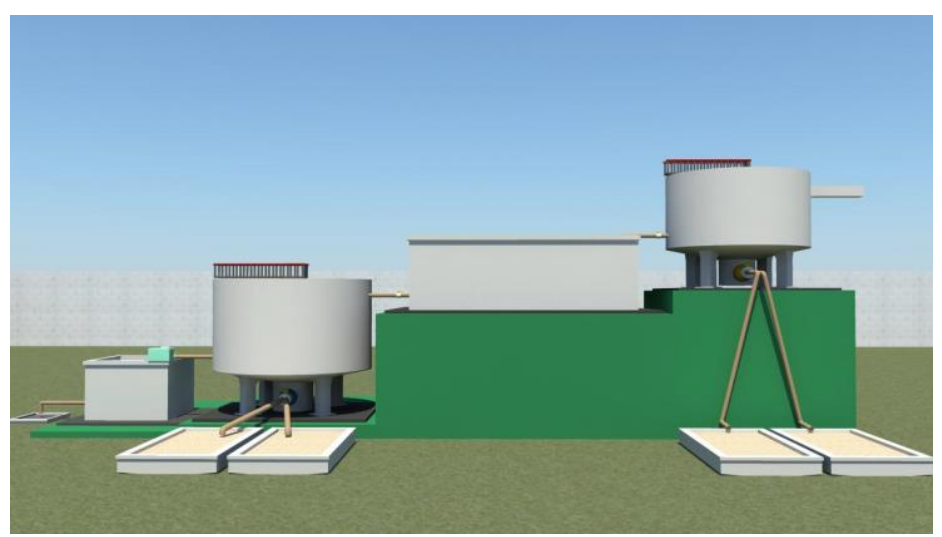

Fuente: Grupo Investigación Facultad Ciencias, 2015

\section{Requerimiento Presupuestario}

TABLA 11: Cotización de la Planta de tratamiento de aguas residuales

\begin{tabular}{cc}
\hline Detalle & Costo $\mathbf{}$ \\
\hline Rejillas & 124,00 \\
Sedimentador primario & 3760,50 \\
Tanque de lodos activados & 13050,00 \\
Sedimentador secundario & 5305,50 \\
Tanque de desinfección & 800,00 \\
Lecho de secado & 6060,00 \\
Accesorios & 2599,90 \\
TOTAL & $\mathbf{3 1 6 9 9 , 4 0}$ \\
\hline
\end{tabular}

Fuente: Grupo Investigación Facultad Ciencias, 2015,

GRAFICO 3. Área total de la Planta de Tratamiento de aguas residuales

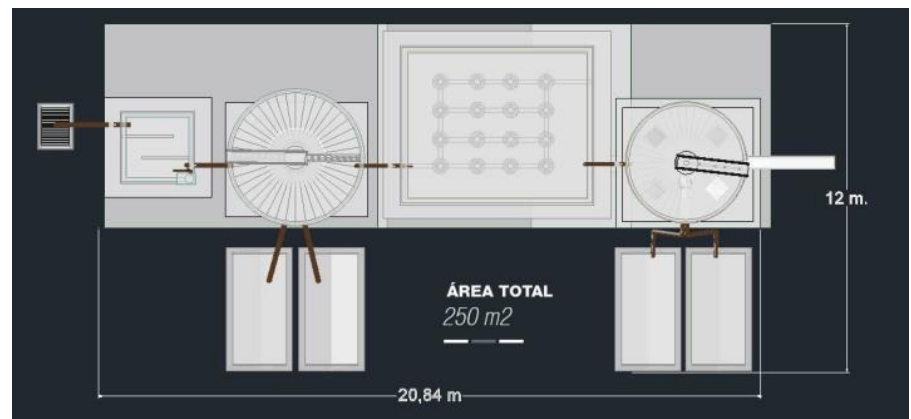

Fuente: Grupo Investigación Facultad Ciencias, 2015 
El costo aproximado de la implementación de la planta es de 31699,40 dólares para la Parroquia Santiago de Calpi, el mismo que necesitará un área Total de $250 \mathrm{~m} 2$ cuyo terreno se encuentra disponible en la Parroquia.

\section{Resultados.}

Las pruebas de tratabilidad realizada a las aguas residuales domiciliarias de la Parroquia de Calpi se basaron en la sedimentación primaria para disminuir la concentración de los sólidos suspendidos, un sistema de aireación de 5 horas, sedimentar el efluente disminuyendo a gran escala la carga de sólidos suspendidos, finalmente debido a la alta presencia de microorganismos en la muestra se consideró agregar Hipoclorito de Calcio al $70 \%$ una cantidad de $0,0015 \mathrm{~g} / \mathrm{L}$ con un tiempo de retención de 30 minutos., el proceso descrito será el que se utilice en la planta de tratamiento de Aguas residuales debido que la eficiencia del mismo es alta en cuanto a la remoción de contaminantes, para que los parámetros se encuentren dentro de los límites permisibles según indica el TULSMA.

El sistema de tratamiento de aguas residuales para la Cabecera Parroquial Santiago de Calpi consta de un sistema de Rejillas con un canal adaptado, Sedimentación primaria, Sistema de Lodos activados, Sedimentación secundaria, Cloración (desinfección) y Lechos de secado de lodos. Cabe recalcar que este sistema está diseñado con mecanismo de gravedad para el mejor funcionamiento de la planta reduciendo costos de accesorios.

La caracterización inicial y final del agua residual se muestra a continuación:

TABLA 12: Caracterización Físico-Química y microbiológica del agua residual

\begin{tabular}{|c|c|c|c|c|}
\hline \multirow[t]{2}{*}{ Parámetros } & \multirow[t]{2}{*}{ Unidad } & \multicolumn{3}{|c|}{ Caracterización Caracterización Valor } \\
\hline & & inicial & final & limite \\
\hline Tensoactivos & $\mathrm{mg} / \mathrm{l}$ & 2,70 & 0,4 & 0,5 \\
\hline Aceites y grasas & $\mathrm{mg} / \mathrm{l}$ & 28 & 0,2 & 0,5 \\
\hline $\begin{array}{l}\text { Demanda } \\
\text { Química de } \\
\text { Oxígeno (DQO) }\end{array}$ & $\mathrm{mg} / \mathrm{l}$ & 368,33 & 59 & 200 \\
\hline $\begin{array}{l}\text { Demanda } \\
\text { Bioquímica de } \\
\text { oxígeno (DBO5) }\end{array}$ & $\mathrm{mg} / \mathrm{l}$ & 287,66 & 37 & 100 \\
\hline $\begin{array}{l}\text { Sólidos } \\
\text { Suspendidos }\end{array}$ & $\mathrm{mg} / \mathrm{l}$ & 195,33 & 2 & 130 \\
\hline $\begin{array}{l}\text { Coliformes } \\
\text { fecales }\end{array}$ & $\begin{array}{l}\mathrm{UFC} / 100 \\
\mathrm{ml}\end{array}$ & $10,15 \times 10^{3}$ & $<2$ & $\begin{array}{l}\text { Remoción } \\
>99 \%\end{array}$ \\
\hline
\end{tabular}

Fuete: Grupo Investigación Facultad Ciencias, 2015

Entre los parámetros físicos se tiene lo sólidos suspendidos con un valor inicial de $195.33 \mathrm{mg} / \mathrm{l}$ y un valor final de $2 \mathrm{mg} / \mathrm{l}$, con un porcentaje de remoción del 98,97 \%, el cual se encuentra dentro del rango permisible. 
Entre los parámetros químicos que con el tratamiento finalmente se encuentran dentro del límite permisible se tiene : Tensoactivos con un valor inicial de 2,7 mg/l y un valor final de $0,4 \mathrm{mg} / \mathrm{l}$, con un porcentaje de remoción del $85 \%$; Aceites y grasas presentan un valor inicial de $28 \mathrm{mg} / \mathrm{l}$, un valor final de $0,2 \mathrm{mg} / \mathrm{l}$ con un porcentaje de remoción del 99,29\%; el DBO5 presenta valores iniciales y finales de 287,66 mg/l y $37 \mathrm{mg} / \mathrm{l}$ respectivamente, con una remoción del 87,13\%; en el DQO se obtuvo un valor inicial de $368,33 \mathrm{mg} / \mathrm{l}$ y un valor final de $59 \mathrm{mg} / \mathrm{l}$ dándonos una remoción del 83,98\%; finalmente el análisis inicial y final con respecto a las coliformes fecales que representan el parámetro microbiológico de análisis se obtuvo valores de $10,15 \times 10^{3}$ UFC/100ml y $<2$ UFC/100ml respectivamente, obteniéndose una remoción mayor al $99,98 \%$.

Todos los parámetros químicos presentados y analizados se compararon con los límites permisibles mencionados en la tabla 9 para descargar a cuerpos de agua dulce, anexo 1, libro IV del TULSMA, para validar los resultados obtenidos una vez aplicado el tratamiento.

\section{Discusión.}

El objetivo principal de esta investigación fue reducir la carga contaminante proveniente de las aguas residuales de la parroquia Santiago de Calpi con el fin de cumplir con lo que estipula el Texto Unificado de Legislación Secundaria Medio Ambiental (TULSMA) libro VI, Anexo 1, tabla 9, debido a que estas aguas residuales son vertidas directamente al río Chibunga sin un previo tratamiento.

Con el diseño propuesto se establece la utilización de un tanque de lodos activados lo que convierte al diseño en un proceso amigable con el medio ambiente.

Los costos de implementación en el caso de que se pusiera en marcha el proceso representaría un inversión económica que justifica la necesidad de la Parroquia Santiago de Calpi de dar tratamiento a las aguas residuales y poder cumplir con la Normativa ambiental vigente en el país; este costo de inversión del sistema de tratamiento de aguas residuales sería de alrededor $\$ 31699,40$.

\section{Conclusiones.}

- Los efluentes que provienen de la Cabecera Parroquial Santiago de Calpi poseen un alto porcentaje contaminante microbiológico y orgánico, y para su respectivo análisis se determinó el caudal de diseño con un valor de 17,125 1/s para la realización del diseño propuesto

- Se realizó la caracterización física, química y microbiológica del agua residual, para determinar los parámetros que se encuentran por encima de los límites máximos permisibles de la Norma ambiental vigente, siendo estos: : Demanda Bioquímica de Oxígeno 287,66 mg/l, Demanda Química de Oxígeno 368,33 
$\mathrm{mg} / \mathrm{l}$, Sólidos Suspendidos 195,33 mg/l, Tensoactivos 2,70 mg/l , Aceites y Grasas $28 \mathrm{mg} / \mathrm{l}$ y Coliformes fecales $10,15 \times 10^{3} \mathrm{UFC} / 100 \mathrm{ml}$.

- El diseño del Sistema de una Planta de Tratamiento de Aguas Residuales consta de los siguientes procesos: Canal de llegada al Sistema de Rejillas, Sedimentador Circular Primario, Tanque de Lodos Activados, Sedimentador Circular Secundario, Tanque de Desinfección, Lechos de Secado.

- Mediante el diseño propuesto se obtuvo la disminución de altos contaminantes presentes en el efluente las variables a consideración para el diseño se caracterizaron los siguientes parámetros obteniéndose los resultados del: Demanda Bioquímica de Oxígeno $37 \mathrm{mg} / \mathrm{L}$, Demanda Química de Oxígeno 59 $\mathrm{mg} / \mathrm{L}$, Sólidos Suspendidos $2 \mathrm{mg} / \mathrm{L}$, Tensoactivos $0,4 \mathrm{mg} / \mathrm{L}$, Aceites y Grasas $0,2 \mathrm{mg} / \mathrm{L}$ y Coliformes fecales $<2 \mathrm{UFC} / 100 \mathrm{ml}$ dichos resultados se encuentran dentro de la Norma del TULSMA 2016 libro IV, anexo 1, tabla 9 límites de descarga a un cuerpo de agua dulce

- Con la caracterización final del agua tratada se validó el tratamiento propuesto con lo que se generó los siguientes porcentajes de remoción: 85\% de Tensoactivos, 99,28\% de Aceites y grasas; $83,98 \%$ de Demanda Química de Oxígeno; 87,13\% de Demanda Bioquímica de Oxígeno; 98,97\% de Sólidos Suspendidos y $99,98 \%$ de Coliformes fecales. El sistema de tratamiento planteado es amigable con el ambiente debido a que utiliza un sistema de lodos activados y que brinda una nueva opción de tratamiento para las aguas residuales, al no aplicar ningún concepto de las plantas de tratamiento comunes.

\section{Referencias bibliográficas.}

Crites, R., \& Tchobanoglous., G. (2000). Tratamiento de Aguas Residuales en Pequeñas Poblaciones., $3^{\mathrm{a}}$ ed., Bogotá - Colombia., McGraw Hill. Pp. 21, 33, 42-44, 46-48.

ECUADOR, INSTITUTO ECUATORIANO DE NORMALIZACION INEN. (2011). Norma Técnica - Ecuatoriana- Requisitos para el agua Potable INEN 1108:2011., 2da. Ed.., Quito - Ecuador.

ECUADOR, INSTITUTO ECUATORIANO DE NORMALIZACION INEN. (2011). Norma Téc-nica - Ecuatoriana- Estudio y Diseño de sistemas de agua potable y disposición de aguas residuales (IEOS). 2da., Quito - Ecuador. Pp. 378

ECUADOR, MINISTERIO DEL AMBIENTE. (2015). Texto Unificado de Legislación Ambien-tal., 2 a ed., Quito - Ecuador., Pp. 330 - 333. 
Geocities, L. (2009). Tratamiento Biológico de Aguas Residuales. [en línea] $15 / 09 / 2015$.

http://www.oocities.org/edrochac/residuales/procesoslodos.htm

Metcalf, \& Eddy. (1995). Ingeniería de Aguas Residuales: Tratamiento, Vertido y Reutiliza-ción., $3^{\mathrm{a}}$ ed., Madrid - España., McGraw-Hill. Pp. 95-102, 508 - 515, $538-551,555-557,605-682$.

Romalho, S. (1996). Tratamiento de Aguas Residuales. $1^{\mathrm{a}}$ ed., Madrid - España. Reverté S.A. Pp 386

Romero, J., Tratamiento de Aguas Residuales., $3^{\text {a }}$ ed., Bogotá - Colombia., Alfaomega., 2002., Pp. $67-71-74-223-706-707$

Russell, D., (2002). Tratamiento de aguas, un enfoque práctico. Barcelona - España. Reverté S.A. pp. 10-11

Orozco, A., (2002). Bioingeniería de Aguas Residuales. Río de Janeiro - Brazil , pp. 408-410.

TULSMA, (2015). Libro VI, Anexo 1, tabla 9 límites de descarga a un cuerpo de agua dulce.

Valdez, C., Vazquez, A. (2003). Ingeniería de los Sistemas De Tratamiento y Disposición De Aguas Residuales., 2a ed., México D F. - México., Fundación ICA, Pp. $3-7$. 


\section{PARA CITAR EL ARTÍCULO INDEXADO.}

Andrade Avalos, M. L., Borja Mayorga, D. F., \& García Veloz, M. J. (2021). Diseño y cotización de una planta de tratamiento de aguas residuales para parroquias rurales del Cantón Riobamba - Provincia de Chimborazo-Ecuador. ConcienciaDigital, 4(2), 198214. https://doi.org/10.33262/concienciadigital.v4i2.1659

\section{Ciencia \\ LDigital}

El artículo que se publica es de exclusiva responsabilidad de los autores y no necesariamente reflejan el pensamiento de la Revista Conciencia Digital.

El artículo queda en propiedad de la revista y, por tanto, su publicación parcial y/o total en otro medio tiene que ser autorizado por el director de la Revista Conciencia Digital.

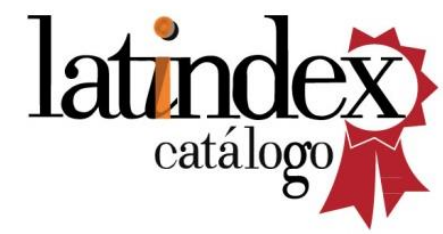

